\title{
Über die Reduktion der Vanadinsäure durch Jod- und Bromwasserstoffsäure und die volumetrische Bestimmung derselben durch Titration in alkalischer Lösung mit Jod. \\ Von
}

\author{
Phimip E. Browning. ${ }^{1}$
}

Die Reduktion der Vanadinsäure, resp. des Pentoxyds zum Tetroxyd durch Einwirkung von Jod und Bromwasserstoffsäure ist schon früher zur volumetrischen Bestimmung des Vanadins angewandt worden. Houverscheit ${ }^{2}$ hat gezeigt, dal's beim Behandeln eines Vanadats mit Kaliumbromid und starker Salzsäure Brom beim Kochen frei wird, und zwar zeigt die Bestimmung desselben durch Einleiten in eine Jodkaliumlösung und Titration des ausgefällten Jods, dals die Reduktion bis zum Tetroxyd gegangen ist. Diese Methode soll sehr zufriedenstellende Resultate ergeben.

FrIEDHerm empfiehlt in einer neueren Abhandlung ${ }^{3}$ diese Methode gleichfalls, und zeigt durch eine sorgfältig ausgeführte Reihe von Bestimmungen, dals das Pentoxyd auch beim Kochen mit Jodkalium und Schwefelsäure in das T'etroxyd übergeführt wird, dass ferner die Reduktion weiter bis zum Trioxyd geführt werden kann, wenn man an Stelle der Schwefelsäure konz. Salzsäure anwendet. Nach beiden Methoden wird das in Freiheit gesetzte Jod in eine Jodkaliumlösung geleitet und in der üblichen Weise bestimmt.

In einer früheren Abhandlung ${ }^{4}$ habe ich gezeigt, dafs man Vanadinsäure bequem bestimmen kann, indem man dieselbe mittels Weinsäure zum Tetroxyd reduziert und dieses im Niederschlag durch direkte Oxydation mit Normaljodlösung bestimmt, nachdem die Lösung abgekühlt und durch Bikarbonat alkalisiert ist. Die Möglichkeit der Ausführung dieser Oxydationsmethode nach der

1 Ins Deutsche übertragen von Edmund Turece.

- Dissertation (Berlin 1890).

3 Ber. deutsch. chem. Ges. 28, 2067.

4 Diese Zeitschr. 7, 158. 
vorausgehenden Reduktion mit Brom- und Jodwasserstoffsäure führte zu den Versuchen, welche in der vorliegenden Arbeit beschrieben werden sollen. Die Vorteile bei dieser Behandlung des Niederschlages sind auf der Hand liegend. Die allgemeine Methode ist ja auch schon bei einer Reihe von Bestimmungen in unserem Laboratorium mit Vorteil angewandt worden. In erster Linie ist der komplizierte Apparat für die Destillation und Aufsammlung des Broms oder Jods nicht notwendig, da ein gewöhnlicher Erlenmeyerkolben für die ganze Reaktion genügt. In Fällen, wo der Destillationsprozefs vorzuziehen ist, kann der Niederschlag zur Kontrole nach unserer neuen Methode behandelt werden und die Resultate nach beiden Methoden werden dann einander ergänzen.

Für die vorliegenden Bestimmungen wurde eine Lösung von Ammoniumvanadat hergestellt und der Gehalt derselben in der Weise bestimmt, dafs man abgemessene Mengen in einen Platintiegel zur Trockne verdampfte und mit einem Tropfen von Salpetersäure erhitzte und zur Wägung brachte. Die zunächst ausgeführte Reduktion mit Jodwasserstoffsäure ging in folgender Weise vor sich: Abgemessene und gewogene Portionen des Ammoniumvanadats wurden in einen Erlenmeyerkolben gebracht, die in der Tabelle angegebenen Mengen von Jodkalium in $10 \%$ iger Lösung und schliefslich $10 \mathrm{ccm}$ einer halb verdünnten Schwefelsäure hinzugefügt. Die Lösung wurde dann gekocht, bis die Dämpfe von Jod nicht mehr sichtbar waren und in dem entweichenden Dampf durch rotes Lackmuspapier ${ }^{1}$ kein freies $J_{o d}$ mehr nachgewiesen werden konnte. Dieser Punkt war erreicht, wenn das Volum der Flüssigkeit bis auf $35 \mathrm{ccm}$ verringert war. Bei Anwendung grofser Mengen von Jodkalium zeigte die Lösung eine grüne Farbe, die auf der Gegenwart von in der Jodwasserstoffsäure gelösten $J_{o d}$ und von blauen Vanadiumtetroxyd beruhte. Bei Anwendung kleiner Mengen von Jodkalium war die Farbe blau. Der Kolben wurde nun von der Flamme entfernt, der Inhalt durch $\ddot{\text { Attzkali }}{ }^{2}$ fast neutralisiert, abgekühlt und die Neutralisation durch Hinzufügen von Natriumbikarbonat im Überschufs vervollständigt. Zugleich wurden einige Tropfen von Weinsäurelösung hinzugefügt, um die Fällung des Tetroxyds zu

1 Gooch und MaR, Amer. Journ. Se. and Arts [3] 39, 400.

${ }^{2}$ Das Atzkali mufste bei diesen Bestimmungen frei von Alkohol sein, da die Lösung später längere Zeit mit Jod in Berührung war. Die Ätzkalilösung war dargestellt durch Mischen von Bikarbonatlösung mit Ätzkalkkali in bestimmtem Verhältnis und Abfiltrieren des Calciumkarbonats. 
verhindern. Die abgekühlte Lösung wurde mit einer Lösung von Jod in Jodkalium in geringem Überschufs versetzt, deren Menge leicht zu bestimmen ist, da die Jodlösung sofort entfärbt wird, wenn sie sich mit der Vanadintetroxydlösung mischt. Sobald die Lösung einen deutlichen Überschuls von Jod enthält, wird der Kolben mit einem mit Paraffin bestrichenen Stopfen verschlossen und 30 Minuten der Ruhe überlassen. Wenn nach 15 Minuten die Farbe des Jods nicht mehr verschwindet, ist die Oxydation beendigt, doch habe ich meistens die Lösungen, um ganz sicher zu gehen, noch einige Zeit länger stehen gelassen. Der Überschuls an Jod wird nun durch eine Normallösung von arseniger Säure, die auf die Jodlösung sorgfältig eingestellt ist, zurücktitriert, Stärkelösung zugefügt und die blaue Farbe mit einigen Tropfen Jodlösung wieder hervorgerufen. Die Menge an Normallösung der arsenigen Säure, welche notwendig war, um die Farbe verschwinden zu lassen, _ist von der gesamten Menge der zur Oxydation des Vanadiums verbrauchten Jodlösung abzuziehen, und es ergiebt sich dann die Menge $J_{o d}$, welche nötig war, um das Vanadium vom Tetroxyd in das Pentoxyd überzuführen. Die Resultate folgen in der untenstehenden Tabelle.

\begin{tabular}{r|c|c|c|c|c}
\hline & $\begin{array}{c}\text { Angewandtes } \\
\mathrm{V}_{2} \mathrm{O}_{5}\end{array}$ & $\begin{array}{c}\text { Gefundenes } \\
\mathrm{V}_{2} \mathrm{O}_{5}\end{array}$ & Fehler & $\begin{array}{c}\text { Angewandtes } \\
\mathrm{KJ}\end{array}$ & $\begin{array}{c}\text { Angewandte } \\
\mathrm{H}_{2} \mathrm{SO} \mathrm{A}_{4} \\
\mathrm{ccm}\end{array}$ \\
\hline \hline 1 & $\mathrm{~g}$ & $\mathrm{~g}$ & & $\mathrm{~g}$ & 1 \\
2 & 0.1699 & 0.1690 & -0.0009 & 1 & 10 \\
3 & 0.1704 & 0.1699 & -0.0005 & 1 & 10 \\
4 & 0.1706 & 0.1700 & -0.0006 & 1 & 10 \\
5 & 0.3613 & 0.1692 & -0.0010 & 1 & 10 \\
6 & 0.1805 & 0.3620 & +0.0007 & 1.5 & 10 \\
7 & 0.3614 & 0.3620 & +0.0002 & 1 & 10 \\
8 & 0.1811 & 0.1814 & +0.0006 & 1.5 & 10 \\
9 & 0.1807 & 0.1815 & +0.0008 & 1 & 10 \\
10 & 0.3613 & 0.3620 & +0.0007 & 1.5 & 10 \\
11 & 0.3679 & 0.3674 & -0.0005 & 1.5 & 10 \\
12 & 0.3612 & 0.3608 & -0.0004 & 1.5 & 10 \\
13 & 0.2893 & 0.2907 & -0.0014 & 1.5 & 10 \\
14 & 0.3456 & 0.3448 & -0.0008 & 1.5 & 10 \\
15 & 0.3453 & 0.3448 & -0.0005 & 1.5 & 10 \\
16 & 0.3907 & 0.3912 & +0.0005 & 2 & 10 \\
17 & 0.3908 & 0.3898 & -0.0010 & 1 & 10 \\
18 & 0.3906 & 0.3921 & +0.0015 & 2 & 10 \\
19 & 0.3909 & 0.3912 & +0.0003 & 1.5 & 10 \\
& & & & & 10 \\
\end{tabular}


Die Bestimmungen sind durchaus zufriedenstellend, da der mittlere Fehler $-0.0002 \mathrm{~g}$ nicht übersteigt. Es muls darauf hingewiesen werden, dals grolse Mengen von Jodkalium Maximalfehler ergaben, die wahrscheinlich darauf beruhen, dafs die Jodwasserstoffsäure Jod zurückhält.

Die Einwirkung der Bromwasserstoffsäure wurde in genau derselben Weise untersucht. Es fand sich, dafs bei einer Volumverminderung der Flüssigkeit auf $25 \mathrm{ccm}$ die blaue Farbe erschien. Die Abwesenheit von freiem Brom im entweichenden Dampf wurde durch ein befeuchtetes Jodkaliumpapier nachgewiesen. Wenn das Kochen nicht bis zu dem Punkt, wo die blaue Farbe der Lösung erscheint, fortgesetzt wird, ergeben sich zu niedrige Resultate, die auf eine unvollständige Reduktion schliefsen lassen. Die Bestimmungen ergaben folgende Zahlen:

\begin{tabular}{r|c|c|c|c|c}
\hline \hline No. & $\begin{array}{c}\text { Angewandtes } \\
\mathrm{V}_{2} \mathrm{O}_{5}\end{array}$ & $\begin{array}{c}\text { Gefundenes } \\
\mathrm{V}_{2} \mathrm{O}_{5}\end{array}$ & Fehler & $\begin{array}{c}\text { Angewandtes } \\
\mathrm{KJ}\end{array}$ & $\begin{array}{c}\text { Angewandte } \\
\mathrm{g} \mathrm{H}_{2} \mathrm{SO} \mathrm{O}_{4} \\
\mathrm{ccm}\end{array}$ \\
\hline \hline & $\mathrm{g}$ & $\mathrm{g}$ & & $\mathrm{g}$ & 10 \\
1 & 0.1890 & 0.1876 & -0.0014 & 1 & 10 \\
2 & 0.1886 & 0.1886 & \pm 0.0000 & 2 & 10 \\
3 & 0.1885 & 0.1882 & -0.0003 & 1 & 10 \\
4 & 0.1885 & 0.1886 & +0.0001 & 1.5 & 10 \\
5 & 0.1881 & 0.1873 & -0.0008 & 1.5 & 10 \\
6 & 0.1896 & 0.1882 & -0.0004 & 2 & 10 \\
7 & 0.3907 & 0.3894 & -0.0013 & 2 & 10 \\
8 & 0.3907 & 0.3903 & -0.0004 & 2 & 10 \\
9 & 0.3907 & 0.3894 & -0.0013 & 2 & 10 \\
10 & 0.3909 & 0.3889 & -00020 & 2 & 10 \\
11 & 0.3911 & 0.3903 & -0.0008 & 1.5 & 10 \\
12 & 0.3902 & 0.3900 & -0.0002 & 2.5 & 10 \\
& & & & &
\end{tabular}

Mittlerer Fehler -0.000 .

The Kent Chemieal Laborutory of Yale University.

Bei der Redaktion eingegangen am 30. Juli 1896. 